\title{
Evaluation of the Accuracy of Various Phenotypic Tests to Detect Oxacillin Resistance in Coagulase-Negative Staphylococci
}

\begin{abstract}
Leandro Reus Rodrigues Perez ${ }^{1,2}$ and Pedro Alves d'Azevedo ${ }^{3}$
${ }^{1}$ Microbiology Unit, Mãe de Deus Hospital; ${ }^{2}$ Postgraduate Program in Pharmaceutical Sciences, Federal University of Rio Grande do Sul (UFRGS); ${ }^{3}$ Postgraduate Program in Medical Sciences, Federal School Foundation of Medical Sciences of Porto Alegre (FFFCMPA); Porto Alegre, RS, Brazil
\end{abstract}

\begin{abstract}
In this study, we determined the accuracy of phenotypic tests (cefoxitin agar dilution, 30 $\mu$ g-cefoxitin and $1 \mu \mathrm{g}$ oxacillin disks) to detect the oxacillin resistance/mecA gene among coagulase-negative staphylococci (CoNS) isolates. The presence of the mecA gene was detected by PCR technique (gold standard). A total of 176 CoNS isolates from blood of hospitalized patients were evaluated. Of these, 138 (78.4\%) harbored the mecA gene. Using 30 $\mu$ g-cefoxitin and $1 \mu$ g-oxacillin disks we obtained 100 and $98.3 \%$ accuracy, respectively. In addition, when cefoxitin was used as marker in an agar dilution method, the higher accuracy $(99.4 \%)$ was established with $8 \mu \mathrm{g}$ cefoxitin per mL breakpoint. Thus, despite of the agar dilution method using cefoxitin as a marker not being standard for this detection, our results suggested that it is an excellent alternative to detect the oxacillin resistance/mecA gene among CoNS isolates.
\end{abstract}

Key-Words: Oxacillin, cefoxitin, CoNS.

Coagulase-negative staphylococci (CoNS), as a group, are a frequently isolated microorganism in nosocomial infections. In Brazil, about 10\%-20\% of bloodstream infections are caused by CoNS and, of the isolates, about 70\%-90\% present resistance to oxacillin [1].

Oxacillin resistance in CoNS is due to the acquisition of the mecA gene that encodes an additional protein, called PBP 2 ', with low affinity to the $\beta$-lactamic antimicrobials $[2,3]$. The accurate determination of oxacillin resistance is necessary for the establishment of therapeutic and surveillance programs [4]. The use of 30 $\mu$ g-cefoxitin disks has been established by the Clinical and Laboratory Standard Institute (CLSI, formerly NCCLS). However, no dilution method using cefoxitin as a marker for oxacillin resistance characterization has been adopted. Thus, the aim of this study was to evaluate the accuracy of phenotypic methods (cefoxitin agar dilution, 30 $\mu \mathrm{g}$ cefoxitin and $1 \mu \mathrm{g}$-oxacillin disks by disk-diffusion) in detecting oxacillin resistance among CoNS isolates and to compare the results with the presence of the mecA gene detected by PCR (gold standard).

\section{Materials and Methods \\ Bacterial Strains and Identification}

From August to December 2004, 176 consecutive CoNS isolates were obtained from blood cultures of patients admitted to three hospitals: Hospital de Clínicas, Irmandade Santa Casa de Misericórdia and Hospital Conceição, in Porto Alegre, RS, Brazil. Only one isolate of each patient was included in the study. The isolates were identified to the species level as previously described [5]. All samples were frozen and stored at $-20^{\circ} \mathrm{C}$ in skim milk with $10 \%$ glycerol.

Received on 3 December 2007; revised 17 March 2008.

Address for correspondence: Dr. Leandro Reus Rodrigues Perez. Laboratory of Clinical Analysis, Faculty of Pharmacy, UFRGS, Ipiranga Avenue 2752/ 303, Porto Alegre, RS, 90610-000, Brazil. Phone: +55 (51) 33085412. Fax: +55 (51) 33085437. E-mail: leandro.reus@gmail.com. This work was partially supported by CNPq, CAPES and FFFCMPA (Brazil).

The Brazilian Journal of Infectious Diseases 2008;12(3):210-212. (C) 2008 by The Brazilian Journal of Infectious Diseases and Contexto Publishing. All rights reserved.

\section{Antimicrobial Susceptibilities Testing}

The susceptibilities to cefoxitin and oxacillin were determined by the disk diffusion method on Mueller-Hinton agar (Oxoid, Basingstoke, UK) plates using bacterial suspensions with turbidity adjusted for 0.5 McFarland standard (Densimat, Rome, Italy), after incubation at $35^{\circ} \mathrm{C}$ for 24h. The results were interpreted according to the CLSI (2006) guidelines.

The minimum inhibitory concentrations (MICs) for cefoxitin were determined by the agar dilution method, according to the CLSI guidelines. Briefly, for each strain, colonies isolated from an overnight growth were transferred to sterile saline; the suspensions were adjusted to a $0.5 \mathrm{McF}$ arland standard and inoculated on Mueller-Hinton agar plates containing 0.25 , $0.5,1,2,4,8,16,32,64,128$ or $256 \mu$ g of cefoxitin per mL using a Steers replicator.

Plates containing Mueller-Hinton agar without antimicrobial were used as controls for bacterial growth.

\section{Polymerase Chain Reaction for mecA Gene}

A polymerase chain reaction (PCR) procedure was used to verify the presence of the mecA gene. Primers (mec $A_{1}$ : 5'TGG CTA TCG TGT CACAAT CG and mecA : 5'-CTG GAA CTT GTT GAG CAG AG) and protocol used were based on the methodology previously mentioned [8].

\section{ROC Curve}

The receiver operator characteristic $(R O C)$ curve was used to determine the best cefoxitin breakpoint to predict oxacillin resistance.

\section{Control Strains}

The quality control of the identification tests was done using Staphylococcus aureus (ATCC 25923), Staphylococcus epidermidis (ATCC 12228), and Staphylococcus hominis (ATCC 27844). The oxacillinsusceptible S. aureus (ATCC 29213) and oxacillin-resistant $S$. aureus (ATCC 33591) isolates were used as controls for antimicrobial susceptibility testing and PCR procedure. 


\section{Results}

A total of 176 CoNS isolates were analyzed. The most frequent species were Staphylococcus epidermidis (120 isolates; $68.2 \%$ ), followed by Staphylococcus haemolyticus (23 isolates; 13.1\%), Staphylococcus capitis (12 isolates; $6.8 \%$ ), Staphylococcus hominis (6 isolates; $3.4 \%$ ), Staphylococcus lugdunensis (5 isolates; 2.8\%) and other CoNS species (10 isolates; $5.7 \%$ ).

One hundred and thirty eight (78.4\%) CoNS isolates were considered oxacillin-resistant based on the presence of the mecA gene detected by PCR. The presence of the mecA gene, using disk-diffusion method, was predicted with $100 \%$ and 98.3\% accuracy for cefoxitin and oxacillin disks, respectively (Table 1). Table 1 summarizes the data showing the sensitivity, specificity, negative and positive predictive values and accuracy for the phenotypic tests applied. Using $1 \mu$ g-oxacillin disk, three isolates (S. epidermidis, S. haemolyticus and $S$. saprophyticus) that did not harbor the mecA gene were oxacillin resistant. According to the agar dilution method, only one isolate (S. epidermidis) that did not harbor the mecA gene was classified as resistant. The others isolates were classified as susceptible to oxacillin when the follow breakpoints were adopted by us: $\geq 8 \mu$ g cefoxitin per $\mathrm{mL}$ and $\leq 4 \mu \mathrm{g}$ cefoxitin per $\mathrm{mL}$ corresponding to resistant (mecA gene present) and susceptible (mecA gene absence) to oxacillin, respectively. Table 2 presents the discrimination among CoNS isolates in comparison to the presence/absence of the mecA gene and its respective cefoxitin MICs.

Figure 1 indicates receiver operating characteristic (ROC) curves for cefoxitin breakpoints when an agar dilution method is used. At this point, our results showed that $8 \mu$ g cefoxitin per $\mathrm{mL}$ is the best breakpoint to discriminate between CoNS isolates that harbored and did not harbor the mecA gene.

\section{Discussion}

The accurate characterization of oxacillin resistance is essential, mainly for correct antimicrobial therapy. Phenotypic tests are important for laboratorial diagnosis. Although molecular methods are considered to be a "gold standard" for the diagnosis of oxacillin resistance, these methods are expensive for many of our laboratories and the use of rapid and accurate phenotypic tests has become an alternative [9].

Since 2004, the CLSI has recommended the use of 30 $\mathrm{gg}$ cefoxitin disk for detection of oxacillin resistance among staphylococci species. Presented in the document M100-S15 are the breakpoints for cefoxitin susceptibility (susceptible $\leq 8 \mu$ g cefoxitin per $\mathrm{mL}$, resistant $\geq 32 \mu$ g cefoxitin per $\mathrm{mL}$ ) although they haven't explicitly specified the mecA gene's presence as the determinant factor for methicillin-resistance. Even thought researches have shown that an agar screening test containing $6 \mu$ g oxacillin per $\mathrm{mL}$ is not efficient towards CoNS [10], comparative studies to asses the agar screening test and disk-diffusion have indicated that agar screening is more sensitive in the detection of methicillin-resistant isolates [11,12]. We have demonstrated that the application of the agar screen test using $4 \mu \mathrm{g}$ cefoxitin per $\mathrm{mL}$ as breakpoint showed $99.4 \%$ accuracy for oxacillin resistance characterization among CoNS [13]. Despite the same accuracy being demonstrated when $4 \mu \mathrm{g}$ oxacillin per $\mathrm{mL}$ was used, cefoxitin agar screen is a feasible method in clinical laboratories and, additionally, it is not necessary to add salt to the culture medium, minimizing errors due to methodological variants.

In this study, cefoxitin breakpoints were evaluated by an agar dilution test, also 30 $\mu$ g-cefoxitin and 1ìg-oxacillin disks, for characterization of oxacillin resistance among CoNS species due to the presence of the mecA gene.

Several studies have showed that the greatest accuracy for oxacillin resistance characterization may be achieved by use of cefoxitin, basically, by the disk-diffusion method $[14,15,16]$. In our study, 98 and $100 \%$ accuracies were obtained

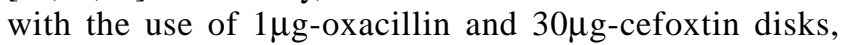

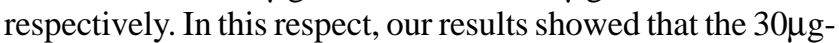
cefoxitin disk presented equal sensitivity but higher specificity than the $1 \mu \mathrm{g}$-oxacillin disk, confirming the better performance of cefoxitin as a marker of this resistance (mecA-mediated resistance).

Fernandes et al. [17] suggest that a breakpoint of $\leq 4 \mu \mathrm{g}$ cefoxitin per $\mathrm{mL}$ is accurate for the determination of oxacillin susceptible $S$. aureus (not harboring mecA gene). The $4 \mu \mathrm{g}$ cefoxitin per $\mathrm{mL}$ breakpoint, in our study, showed less accuracy than an $8 \mu$ g cefoxitin per $\mathrm{mL}$ breakpoint $(97.1 \%$ vs. 99.4\%) (Figure 1).

The study described values of $\geq 8 \mu \mathrm{g}$ cefoxitin per $\mathrm{mL}$ and $\leq 4 \mu$ g cefoxitin per $\mathrm{mL}$ as the best breakpoints for characterization of oxacillin -resistant and -susceptible isolates, respectively. Using $8 \mu \mathrm{g}$ cefoxitin per $\mathrm{mL}$, only one $S$. epidermidis isolate (with MIC of $16 \mu$ g cefoxitin per $\mathrm{mL}$ ) was mischaracterized as resistant (Table 2). This breakpoint was shown to be highly reliable in the discrimination of isolates harboring the mecA gene. On the other hand, discrepancies can be observed between isolates harboring and those not harboring the mecA gene because this event (resistance without the presence of the mecA gene) may be associated with the hyperproduction of $\beta$-lactamase in these isolates or mutations in PBP's other than PBP 2'. Swenson et al. showed that a cefoxitin test can be used alone to predict mecA-mediated resistance, when these tests were applied for the characterization of this resistance among $S$. aureus isolates $[18,19]$. However, despite the excellent concordance between cefoxitin tests and the mecA-gene, non-mecA-mediated resistance may be underestimated [18].

In conclusion, the $30 \mu \mathrm{g}$-cefoxitin disk was shown to be more specific than the $1 \mu \mathrm{g}$-oxacillin disk in the prediction of oxacillin resistance due to the mecA gene among CoNS. Also, our results suggested that $8 \mu$ g cefoxitin per $\mathrm{mL}$ is the more accurate breakpoint (99.4\%) when an agar dilution method is applied, despite oxacillin resistance (mecA gene) detection not being actually done by it.

\section{References}

1. Sader H.S., Jones R.N., Gales A.C., et al. SENTRY antimicrobial surveillance program report: Latin American and Brazilian results for 1997 through 2001. Braz J Infect Dis 2004;8:25-79.

2. Chambers H.F. Penicillin-binding protein-mediated resistance in pneumococci and staphylococci. J Infect Dis 1999;179:353-9. 
Table 1. Sensitivity, specificity, negative and positive predictive values and accuracy for different phenotypic tests applied to predict the presence of the mecA gene considering the PCR procedure as gold standard.

\begin{tabular}{|c|c|c|c|c|c|}
\hline Phenotypic tests & Sens $^{\mathrm{c}}(\%)$ & $\operatorname{Spec}^{c}(\%)$ & NPV $^{c}(\%)$ & $\mathrm{PPV}^{\mathrm{c}}(\%)$ & Accur $(\%)$ \\
\hline $1 \mu \mathrm{g}$-oxacillin disk $\mathrm{k}^{\mathrm{a}}$ & 100 & 92.1 & 100 & 97.9 & 98.3 \\
\hline 30ug-cefoxitin disk ${ }^{\mathrm{a}}$ & 100 & 100 & 100 & 100 & 100 \\
\hline $8 \mu \mathrm{g}$ cefoxitin per $\mathrm{mL}^{\mathrm{b}}$ & 100 & 97.4 & 100 & 99.3 & 99.4 \\
\hline $4 \mu g$ cefoxitin per $m L^{b}$ & 100 & 86.8 & 100 & 96.5 & 97.1 \\
\hline
\end{tabular}

a Using a disk-diffusion method; ${ }^{b}$ Using an agar dilution method; 'Sens, sensitivity; Spec, specificity; NPV, negative predictive value; PPV, positive predictive value and Accur, accuracy.

Table 2. Presence of the mecA gene and cefoxitin minimum inhibitory concentration among CoNS isolates analyzed.

\begin{tabular}{|c|c|c|c|c|c|c|c|c|c|c|c|c|}
\hline \multirow[b]{2}{*}{$\mathrm{N}^{\circ}$ of isolates } & \multirow[b]{2}{*}{$\mathbf{P C R}^{\mathbf{b}}$} & \multicolumn{11}{|c|}{ Number of isolates with cefoxitin $\mathrm{MIC}^{\mathrm{a}}(\mu \mathrm{g}$ cefoxitin per $\mathrm{mL}$ ) } \\
\hline & & 0.25 & 0.5 & 1 & 2 & 4 & 8 & 16 & 32 & 64 & 128 & 256 \\
\hline 38 & mecA - & 2 & 16 & 15 & 4 & & 1 & & & & & \\
\hline 138 & mecA + & & & & & & 24 & 42 & 14 & 8 & 19 & 31 \\
\hline
\end{tabular}

aCefoxitin MIC determined by an agar dilution method; ${ }^{\mathrm{b}} \mathrm{mec} \mathrm{A}$ gene was detected by PCR; $m e c \mathrm{~A}+=$ gene detected; $m e c \mathrm{~A}-=$ gene not detected; Total number of isolates $(\mathrm{n})=176$; the shaded numbers of isolates in the table were correctly categorized as resistant $\left(m e c \mathrm{~A}^{+}\right)$and susceptible (mecA-), respectively, when $\geq 8 \mu \mathrm{g}$ cefoxitin per $\mathrm{mL}$ and $\leq 4 \mu \mathrm{g}$ cefoxitin per $\mathrm{mL}$ breakpoints were used.

Figure 1. $R O C$ curve established for the cefoxitin breakpoints by an agar dilution method. The best performance including sensitivity and specificity were achieved in $8 \mu$ g cefoxitin per $\mathrm{mL}$.

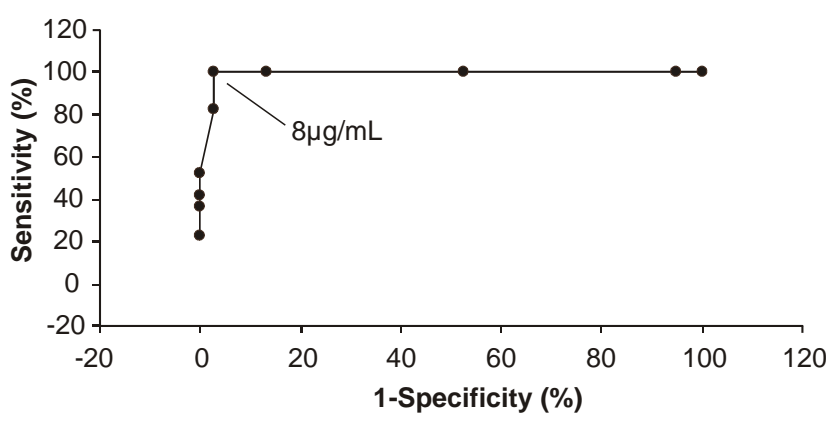

3. Fuda C., Suvorov M., Vakulenko S.B., Mobashery S. The basis for resistance to beta-lactam antibiotics by penicillin-binding protein 2a of methicillin-resistant Staphylococcus aureus. J Biol Chem 2004;279:40802-6.

4. Huebner J., Goldmann D.A. Coagulase-negative staphylococci: role as pathogens. Annu Rev Med 1999;50:223-36.

5. Bannerman T. Staphylococcus, Micrococcus and other catalasepositive cocci that grow aerobically. In: Murray P.R., Baron E.J., Jorgensen J.H., Pfaller M.A., Yolken R.H. (eds). Manual of Clinical Microbiology. American Society of Microbiology, Washington, DC, pp 384-404, 2003.

6. Clinical and Laboratory Standard Institute - CLSI. Methods for dilution antimicrobial susceptibility tests for bacteria that grow aerobically. Approved standard $15^{\text {th }}$ CLSI document M7-A7. Wayne, PA: CSLI/(NCCLS), 2006.

7. Clinical and Laboratory Standard Institute - CLSI. Performance standards for antimicrobial susceptibility testing. $15^{\text {th }}$ informational supplement. M100-S15. Wayne, PA: CSLI/(NCCLS), 2006.

8. Vannuffel P., Laterre P.F., Bouyer M., et al. Rapid and specific molecular identification of methicillin-resistant Staphylococcus aureus in endotracheal aspirates from mechanically ventilated patients. J Clin Microbiol 1998;36:2366-8.
9. Ferreira R.B., Iorio N.L., Malvar K.L., et al. Coagulase-negative staphylococci: comparison of phenotypic and genotypic oxacillin susceptibility tests and evaluation of the agar screening test by using different concentrations of oxacillin. J Clin Microbiol 2003;41:3609-14.

10. Tenover F.C., Jones R.N., Swenson J.M., et al. Methods for improved detection of oxacillin resistance in coagulase-negative staphylococci: results of a multicenter study. J Clin Microbiol 1999;37:4051-8.

11. Kohner P., Uhl J., Kolbert C., et al. Comparison of susceptibility testing methods with mecA gene analysis for determining oxacillin (methicillin) resistance in clinical isolates of Staphylococcus aureus and coagulase-negative Staphylococcus spp. J Clin Microbiol 1999;37:2952-61.

12. York M.K., Gibbs L., Chehab F., Brooks G.F. Comparison of PCR detection of mecA with standard susceptibility testing methods to determine methicillin resistance in coagulase-negative staphylococci. J Clin Microbiol 1996;34:249-53.

13. Perez L.R.R., Antunes A.L., Barth A.L., d'Azevedo P.A. Variations of agar screen tests for detection of methicillin resistance in staphylococci: focus on cefoxitin. Eur J Clin Microbiol Infect Dis 2007;26:267-70.

14. Pottumarthy S., Fritsche T.R., Jones R.N. Evaluation of alternative disk diffusion methods for detecting mecA-mediated oxacillin resistance in an international collection of staphylococci: validation report from the SENTRY Antimicrobial Surveillance Program. Diagn Microbiol Infect Dis 2005;51:57-62.

15. Skov R., Smyth R., Larsen A.R., et al. Evaluation of cefoxitin 5 and 10 microg discs for the detection of methicillin resistance in staphylococci. J Antimicrob Chemother 2005;55:157-61.

16. Zhu L.X., Zhang Z.W., Wang C., et al. Evaluation of the CLSI cefoxitin 30-microg disk-diffusion method for detecting methicillin resistance in staphylococci. Clin Microbiol Infect 2006; $12: 1039-42$.

17. Fernandes C.J., Fernandes L.A., Collignon P. Cefoxitin resistance as a surrogate marker for the detection of methicillin-resistant Staphylococcus aureus. J Antimicrob Chemother 2005;55:506-10.

18. Swenson J.M., Lonsway D., McAllister S., et al. Detection of mecAmediated resistance using reference and commercial testing methods in a collection of Staphylococcus aureus expressing borderline oxacillin MICs. Diagn Microbiol Infect Dis 2007;58:33-39.

19. Swenson J.M., Tenover F.C. Cefoxitin Disk Study Group. Results of disk diffusion testing with cefoxitin correlate with presence of mecA in Staphylococcus spp. J Clin Microbiol 2005;43:3818-23. 